\title{
Negative and matrix-dependent effects of dispersal corridors in an experimental metacommunity
}

\author{
JENS Åström ${ }^{1,3}$ AND TOMAS PÄRT ${ }^{2}$ \\ ${ }^{1}$ Norwegian Institute for Nature Research, P.O. Box 5685 Sluppen, NO-7485 Trondheim, Norway \\ ${ }^{2}$ Department of Ecology, Swedish University of Agricultural Sciences, Box 7044, 75007 Uppsala, Sweden
}

Abstract. Negative effects of habitat fragmentation are well-known phenomena in ecology, and the use of corridors is one suggested remedy to ameliorate community disassembly. Most experiments of landscape structure, however, do not consider matrix quality and environmental conditions, despite their potential to affect both landscape permeability and population densities. For the first time in fragmented landscapes, we simultaneously investigated the effects of local disturbance, dispersal corridors, matrix quality, and environmental stress, as well as their interactions, on species richness and abundance. We used a natural micro-ecosystem of bryophyte patches with microarthropods as a model system. Contrary to expectations, there were no negative responses to fragmentation of large continuous habitats. Surprisingly, connecting fragmented patches with dispersal corridors had negative effects on the abundance and species richness of the most species-rich group, oribatid mites. It had also negative effects on the abundance of collembolans and predatory mites in landscapes with complex matrix, which was also generally detrimental. Environmental stress was detrimental for all taxa but interacted with matrix type for oribatid species richness and juvenile oribatid abundance. Our results indicate that interactions can strongly alter community responses to common explanatory factors such as fragmentation, disturbance, connectivity, and environmental quality. Future studies of metacommunity dynamics need to consider the potential for such interactions in order to produce robust predictions of spatially structured landscapes.

Key words: bryophyte; dispersal corridor; disturbance; environmental quality; fragmentation; matrix quality; metacommunity; microarthropods; micro-ecosystem; oribatid mites.

\section{INTRODUCTION}

The effects of landscape structure on biodiversity and community composition is a current topic in theoretical as well as empirical ecological research (Lindenmayer and Fischer 2006). One reason for this interest is the ongoing exploitation of natural environments, which leaves habitats increasingly fragmented. Another reason is the recent development of metacommunity theory that compares the importance of local and regional processes (Leibold et al. 2004, Holyoak et al. 2005, Gravel et al. 2010). Still, mechanistic metacommunity models that generate detailed predictions are seldom used in the prescription of conservation and management strategies. This is partly because natural systems rarely conform to a single metacommunity concept (Cottenie 2005, Holyoak et al. 2005) and partly because outcomes in specific cases rely on the interplay of trophic interactions, spatial structure, disturbance, and environmental quality. Studies on how these factors interact is therefore of importance in connecting the theoretical models with natural situations. An example of such interactions is

Manuscript received 5 October 2011; revised 6 August 2012; accepted 16 August 2012. Corresponding Editor: D. S. Srivastava.

${ }^{3}$ E-mail: jens.astrom@nina.no that fragmented habitats (smaller, disconnected habitats) might be more affected by disturbance than continuous habitats, because recolonization is less likely in isolated habitats (Starzomski and Srivastava 2007). Landscape structure has also been found to influence disturbance-sensitive species more than tolerant species (Vanschoenwinkel et al. 2010).

Although the matrix (the environment between habitat patches) traditionally has been seen as an inert canvas in metapopulation theory, increased attention has been given to the importance of matrix quality in fragmented and spatially heterogeneous environments (e.g., Öckinger and Smith 2008, Perfecto and Vandermeer 2010, Öckinger et al. 2011). Contrary to simplified descriptions of patchy environments, organisms do occasionally occupy and disperse through the matrix. Dispersal corridors (connecting strips of habitat) may increase effective population sizes (Hanski 1999, Haddad and Tewksbury 2006) and create rescue effects through inter-patch movement (Brown and KodricBrown 1977), thereby reducing the extinction risk of populations. On the other hand, habitat corridors as a management action has been criticized due to possibly increased predation pressure, as well as negative edge effects (Simberloff and Cox 1987), which potentially could be exacerbated by a harsh matrix. It can be 
expected that the importance of corridors for maintaining diversity and community function should increase as matrix quality and permeability deteriorates, rendering connecting corridors more important. However, the end results of the creation of corridors rely on the net benefit of several potentially opposing mechanisms.

Harsh environmental conditions have the potential to either increase or decrease the relative importance of structural landscape elements, such as dispersal corridors. First, increased environmental stress could potentially enhance species sorting (Leibold et al. 2004), because this creates a less permissive environmental filter, and thereby decrease the relative importance of regional processes. This might not affect the number of dispersers through corridors, but it would decrease their relative importance in the composition of the metacommunity. On the other hand, high environmental stress could lead to fluctuating and smaller population sizes, thus increasing the importance of inter-patch dispersal. This could manifest as increased importance of corridors, because this reduces population abundances and leads to local population extinctions and, thereby, a larger need for rescue (Brown and KodricBrown 1977, Hanski 1999). An hypothesized interaction between landscape structure and environmental stress is therefore a potential explanation for the discrepancy between similar studies that either found negative effects of fragmentation (Gilbert et al. 1998, Gonzalez and Chaneton 2002), or did not (Hoyle and Gilbert 2004, Hoyle and Harborne 2005). Hoyle and Gilbert (2004) indeed speculated that the lack of fragmentation effect in their case could be due to favorable environmental conditions limiting the need for rescue effects.

Because generation time and dispersal ability often vary between trophic levels, it can be assumed that different trophic levels will react to fragmentation differently. For example, predators may be more heavily affected by fragmentation due to their sensitivity to habitat size (Holt et al. 1999). Conversely, if predators have high enough dispersal capacity, they may be unaffected by fragmentation, while it still affects their prey. Local predation pressure can therefore both decrease and increase in response to fragmentation, depending on the dispersal capacity of the predators (Howeth and Leibold 2010).

This discussion suggests that the spatial structure of a landscape can interact with several factors. The effect of spatial structure could be dependent on (at least) disturbance, species identity (governing traits such as trophic position or dispersal ability), matrix quality, and environment. No study so far has simultaneously investigated the effects of all these factors on local communities. Here, we analyze the importance of habitat loss and connectivity, and specifically test for interactions between disturbance, dispersal corridors, matrix quality, and environmental stress. We performed an experiment using 62 replicate landscapes with over 200 individual patches, containing in total more than
500000 animals, in which we could manipulate all of the variables of interest in a controlled manner. The time period allowed for significant dispersal between habitat patches but was short enough to limit the importance of reproduction. Thus, this study does not capture longterm, and possibly separate, effects of fragmentation. We expected all but the most dispersive species (collembolans) to be negatively affected by fragmentation (Åström and Bengtsson 2011). We hypothesized that the negative effects of fragmentation should be exacerbated by environmental stress and low matrix quality. In similar experiments, habitat corridors have been shown to alleviate negative population trends and extinctions due to fragmentation, through rescue effects (Gonzalez et al. 1998). We expected habitat corridors to increase abundance and species richness more as environmental stress increases, as well as homogenize the abundances and morphospecies richness between disturbed and undisturbed patches. Similarly, we expected that the positive effects of habitat corridors would be greater in landscapes with low matrix quality, due to greater differences in dispersal rates in corridors relative to in the matrix.

\section{Material And Methods Study system}

We used moss carpets and their naturally occurring microarthropod communities as raw material to create experimental landscapes (Gonzalez et al. 1998, Srivastava 2004). The moss consisted mainly of Rachomitrium spp. and was collected from a rock surface in a managed forest in Hållnäs, Sweden $\left(60.553^{\circ} \mathrm{N}, 17.868^{\circ} \mathrm{E}\right)$, dominated by pine (Pinus sylvestris) and spruce (Picea abies). Earlier studies (Rantalainen et al. 2006, Åström and Bengtsson 2011) have suggested possible longdistance dispersal of microarthropods from surrounding habitats, leading us to locate the present experiment on a rooftop, $\sim 3 \mathrm{~m}$ above ground level, in order to limit the immigration of individuals from the outside.

We constructed 96 replicate landscapes for the study (Fig. 1). Each experimental landscape, with or without dispersal corridors, consisted of four habitat patches of which one was heavily disturbed (i.e., defaunated) at the start of the experiment and the rest were untreated. After the initial defaunation, the disturbed patches experienced environmental conditions similar to those of the non-disturbed patches. Patches were cut out from the collected moss material by a circular metal punch with an area of $100 \mathrm{~cm}^{2}$. The corridors used for interlinking the patches were $2 \times 7 \mathrm{~cm}$ wide and made from the same material, creating a complete landscape of $\sim 30 \times 30 \mathrm{~cm}$. The corridor fragments were defaunated at the start of the experiment. Landscapes without corridors had the equivalent amount of defaunated habitat added to them to keep the overall habitat area constant between treatments. The moss landscapes were placed on $60 \times 60 \mathrm{~cm}$ plywood surfaces 


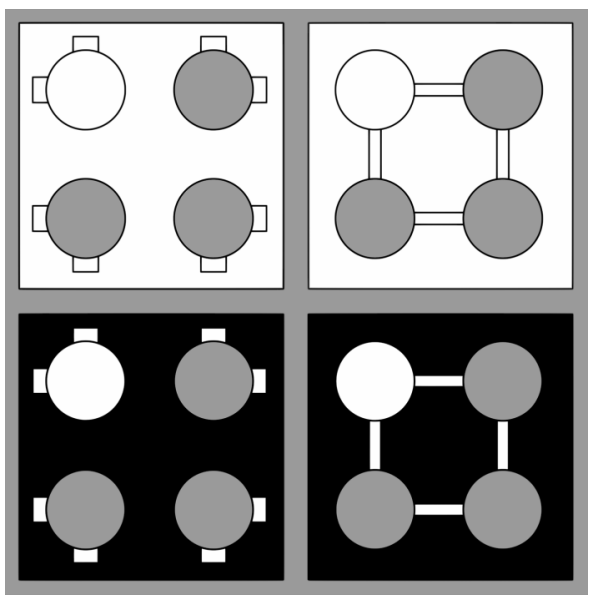

FIG. 1. Experimental design showing the different experimental landscapes, consisting of circular $100-\mathrm{cm}^{2}$ habitat patches and $2 \times 7 \mathrm{~cm}$ dispersal corridors created from larger patches of moss (bryophytes). Study organisms belonged to the microarthropod groups mites (Acari) and springtails (Collembola), which occurred naturally within the source patches of moss. Each landscape contained four habitat patches (circles) that were either isolated or linked by corridors. Patches not linked by dispersal corridors had the equivalent amount of habitat added to them. White patches are defaunated habitat, gray patches are undefaunated habitat; white and black squares are plywood and gravel matrix, respectively. In addition to the corridor and matrix treatment, half of the landscapes also had a mesh roof (not shown) and were subjected to regular watering, constituting the environmental quality treatment.

surrounded by coarse, round gravel, $1-2 \mathrm{~cm}$ in diameter. Replicate landscapes were placed $\sim 1 \mathrm{~m}$ apart.

Matrix quality was manipulated by contour-sawing the plywood sheets at the border of the moss fragments, leading to the moss habitats being directly surrounded either by gravel or by the plywood surface. The gravel matrix was expected to be a significantly worse matrix habitat, in terms of both permeability and microclimate, because the gravel bed constitutes a considerable topography relative to the species involved and can reach high temperatures during summer days due to solar radiation. Environmental stress was manipulated by placing a roof of mosquito netting on top of the experimental landscapes and applying regular watering. The mesh roofs limited both air movement and solar influx, which, in combination with watering, reduced the risk of desiccation. Under natural conditions, moss patches located on hard surfaces such as rocks, or in this case plywood, experience prolonged periods of desiccation during the summer months. Drought has been found to significantly decrease the abundance and species richness of soil microarthropods, during both longer and shorter time periods (Lindberg et al. 2002, Starzomski and Srivastava 2007). The experiment ran for 103 days during July-October 2007, a time period that did not allow for significant reproduction (because most species were expected to complete one or fewer reproductive cycles), but was still long enough for considerable dispersal to occur (Luxton 1981, Siepel 1994, Walter and Proctor 1999).

The experiment was a full factorial design with 12 replicates of each treatment combination: two levels of matrix quality, two levels of environmental stress, and two levels of connectivity. However, due to interference from corvid birds, a considerable amount of the landscapes without roofs had to be discarded, making the total sample size 239 patches and 62 landscapes (for implications of this, see Methods: Analyses). In addition to the fragmented landscapes, six continuous $60 \times 60 \mathrm{~cm}$ areas of moss were kept for the entire experimental period as a reference of unfragmented habitat. These carpets of moss received the "high-quality" matrix and "low environmental stress" treatment and were subsampled (27 samples) at the end of the experiment by the same metal punch used to create the fragmented landscapes.

Sample extraction was done in a modified Tullgren funnel, where animals move along a heat and light gradient down into a collecting jar. Animals were extracted during 72 hours and conserved in $70 \%$ ethyl alcohol. Although extraction efficiency is not $100 \%$, it should have been approximately equal for all patches. The initial defaunation of patches and corridors was done by repeated extractions in Tullgren funnels with intervening rewetting and freezing, which has been shown to be an effective defaunation process (Åström and Bengtsson 2011; N. Lindberg, unpublished data), based on checking the number of individuals in defaunated patches that had been left isolated from dispersal sources for a year (visually estimated to neglible amounts). The animals were collected and sorted into taxonomic groups of varying resolution. Adult oribatid mites were identified mostly to family level, and are called morphospecies hereafter. Other identified groups were: juvenile oribatid mites, collembolans, adult large prostigmatid mites, adult large mesostigmatid mites (mostly Zerconidae), and small/ juvenile mesostigmatid/prostigmatid mites, spiders, and other arthropods. The larger prostigmatids consisted mainly of families Bdellidae and Rhagidiidae and were combined with the large mesostigmatid mites to form a functional group of predators. Only the results for adult oribatids, juvenile oribatids, oribatid morphospecies richness, predators, and collembolans are reported here, due to low numbers of other groups. The oribatids mainly graze on detritus and fungi (Remén et al. 2010); as adults, they have a hardened exoskeleton that protects against predation and, to some extent, drought (Hutson 1980, Walter and Proctor 1999). The collembolans are mainly detritivores, with low drought tolerance, and relatively high reproductive rates (Lindberg and Bengtsson 2006, Auclerc et al. 2009). Explicit data on dispersal capabilities under the present conditions are largely lacking, but based on earlier studies (Åström and Bengtsson 2011), trophic relationships, and 
morphological characteristics (body size and length of legs), we expected that dispersal capabilities would be generally distributed as oribatid mites $<$ predators $<$ collembolans, although some overlap could be expected.

\section{Analyses}

In all statistical analyses, abundances and species richness of the individual patches were analyzed using generalized mixed models with Poisson-lognormal errors and log link. Overdispersion was handled by incorporating a random effect at the individual sample level, hence, Poisson-lognormal (Hinde 1982, Elston et al. 2001), except for morphospecies richness, which was not overdispersed. Models were fitted by lme4 (Bates and Maechler 2010) in R (R Development Core Team 2010). Remaining overdispersion was inspected by comparing the sum of the squared Pearson residuals to a $\chi^{2}$ distribution. The mixed models that were used are generally robust to unbalanced data sets (Venables et al. 1998), and because the interference from the birds was apparently random, we expect the loss of experimental units to only affect the power of the tests, specifically involving the environmental treatment, and not to bias the estimates. A table of the remaining experimental units can be found in the Appendix. A priori tests of the effect of fragmentation were made by including continuous/fragmented as a fixed effect, comparing the samples from the continuous reference moss carpets to the patches in the experimental landscapes. Only undefaunated patches located in a high-quality matrix (plywood) and with low environmental stress (roof) were used for this comparison (72 samples). Fragmentation effects were tested by likelihood ratio tests and were compared to a $\chi^{2}$ distribution. Reported effect sizes of this comparison are from model estimates.

Abundance and morphospecies richness within the fragmented landscapes were analyzed using a generalized mixed model with the same details as previously described. Fixed effects were (1) disturbance by defaunation, (2) presence of corridors, (3) matrix quality, and (4) environmental stress. Potential second-order interactions were also included. Third-order interactions did not improve model fits as measured by $\mathrm{AIC}_{\mathrm{c}}$ and were not included in the final analyses. The logarithm of the patch dry mass was included as an offset, scaling the intercept of the models to numbers per gram dry mass. Replicate landscapes were included as a random effect (62 levels), as well as individual sample (239 levels), to account for overdispersion.

Because there are a multitude of methods for inference and model selection of generalized mixed models (Bolker et al. 2009), and each has its merits and pitfalls, we compared the results from several methods: model-averaging by $\mathrm{AIC}_{\mathrm{c}}$, backwards selection, and selection by best $\mathrm{AIC}_{\mathrm{c}}$. Generally, all approaches aligned well with each other. Backwards selection occasionally dropped terms that were slightly above the 0.05 threshold, but which explained the data well enough to be included in the models with best $\mathrm{AIC}_{\mathrm{c}}$. All reported effect sizes of the fragmented patches are from comparisons of predicted means on a full, balanced (orthogonal) data set, based on model-averaged estimates, by predicting also to the units that were destroyed by birds. Model-averaging was based on $\mathrm{AIC}_{\mathrm{c}}$ and included models making up the top $90 \%$ of cumulative weights (see Appendix for parameter estimates). All reported effects stood out clearly in the analyses (large estimated effect size, high relative importance), unless stated specifically as tendencies.

Diversity was partitioned into $\alpha$-, $\beta$-, and $\gamma$-components per replicate landscape and the dependence of the relative size of these components on the treatments was investigated by randomization tests (Anderson 2001). This was done both for species richness and Shannon diversity. $\beta$-diversity measured as Bray-Curtis dissimilarity within replicate landscapes was tested similarly. However, analysis of the partitioning of $\alpha-, \beta$-, and $\gamma$ diversity did not indicate a dependence of the relative proportions of diversity components on any experimental treatments, and the results are therefore not further reported. A possible reason for the lack of effect on $\alpha$-, $\beta$-, and $\gamma$-diversity components is lack of power, because the randomization procedure only had four patches in each landscape to shuffle. In addition, we compared the responses to the treatments for the different morphospecies of oribatids. At least the species making up $95 \%$ of the total abundance reacted virtually in unison to the experimental treatments. We therefore do not further report species-specific results. Throughout the paper, we analyze abundances and species richness at the local patch level.

In total, 517851 animals were counted. There were 56115 adult oribatids of 20 morphospecies, 223767 juvenile oribatids, 210073 collembolans, and 2016 predators. Remaining individuals (25 880) were juvenile or small species of prostigmatids and mesostigmatids and a few spiders that were not included in the analysis for practical reasons.

\section{Results}

\section{Fragmentation}

Before analyzing the different treatments of the fragmented landscapes, we first investigated the importance of fragmentation per se, by comparing continuous moss vs. a subset of the fragmented landscapes, treated in a similar way (see Methods for details). The effects of fragmentation (continuous vs. fragmented habitat) varied between groups of species (Table 1). Contrary to expectations, there was a tendency for the nonpredatory groups to be more abundant and species rich (oribatid morphospecies) in the fragmented landscapes as compared to the continuous habitat, whereas predators did not show such a tendency. However, this fragmentation effect was only statistically significant for oribatid species richness (at $\alpha=0.05$ ). 
TABle 1. Comparison between continuous habitat and fragmented habitat for experimental micro-ecosystems with microarthropods.

\begin{tabular}{lccc}
\hline \hline \multirow{2}{*}{\multicolumn{1}{c}{ Response }} & \multicolumn{2}{c}{ Fragmented vs. continuous } \\
\cline { 2 - 4 } & Estimate & SE & $P$ \\
\hline Adult oribatid mites & 1.28 & 1.42 & 0.491 \\
Juvenile oribatid mites & 1.43 & 1.19 & 0.055 \\
Oribatid species richness & 1.26 & 1.10 & 0.023 \\
Collembola abundance & 1.46 & 1.59 & 0.414 \\
Predator abundance & 0.97 & 1.35 & 0.919 \\
\hline
\end{tabular}

Note: Estimates are back-transformed; i.e., an estimate of 1.28 means that fragmented habitat contained $128 \%$ of adult oribatids relative to continuous habitat, and so forth.

\section{Main effects in fragmented landscapes}

The treatments of the fragmented moss landscapes, i.e., disturbance, corridors, matrix quality, and environmental condition, all strongly influenced at least parts of the microarthropod community. The results are summarized for each of the five studied groups in Table 2 and Figs. 2 and 3. In general, results were as follows.

Disturbed (i.e., defaunated), unconnected patches had lower average abundance than non-disturbed patches for all species groups. The lowest level of recolonization was observed among predators (44\% of that in the surrounding undisturbed patches). Collembolans and juvenile and adult oribatids, on the other hand, recolonized to $\sim 75 \%$ of the abundances in surrounding patches.

Landscapes of low matrix quality had lower average abundances than landscapes with high matrix quality for all species groups (roughly $18-45 \%$ reduction among oribatids; but see juvenile oribatids in landscapes with high environmental stress [Fig. 2] and $\sim 66 \%$ and $63 \%$ reduction among collembolans and predators, respectively). Oribatid morphospecies richness was reduced by $\sim 8 \%$ in landscapes of low matrix quality (Fig. 3).

Landscapes with high environmental stress had lower average abundances than those with low environmental stress (i.e., shade and extra water) for most groups of species (a decrease in abundance of $\sim 34 \%$ for adult oribatids, $80 \%$ for collembolans, and $40 \%$ for predators).
In contrast to expectations, the addition of corridors had no effect or negative effects on the abundance of most species (Fig. 2). This was most evident for adult oribatids, which showed reduced abundances of $\sim 40 \%$ in the presence of corridors. This negative effect was consistent for all oribatid species abundant enough to influence the total adult oribatid abundance (data not shown). Collembolans and predators only displayed a reduction due to corridors in landscapes with gravel matrix. There was no clear impact of corridors on juvenile oribatid abundance, but there was a tendency for lower oribatid morphospecies richness in landscapes with corridors ( $\sim 7 \%$ fewer species). However, the effects of corridors were contingent on other treatments for several measurements, interactions that we now address.

\section{Corridor interaction effects}

Although there was generally lower abundance in disturbed patches than in undisturbed patches at the end of the experiment, the presence of corridors diminished this difference for adult oribatids and predators (corridor $\times$ disturbance interaction). However, the specifics differed between the two taxa. Although corridors mainly increased the predator abundance in disturbed habitats, the result for oribatids was rather reduced abundances in the undisturbed patches (Fig. 2). No other groups of species showed interactions between disturbance and corridors.

We also found that the response to corridors was dependent on matrix quality for some groups of species. In landscapes with gravel matrix, presence of corridors decreased the abundance of collembolans (39\% reduction) and predators (51\% reduction), whereas this negative effect of corridors disappeared in landscapes with plywood matrix (Fig. 2). In landscapes with plywood matrix, corridors actually had a tendency to increase predator abundance ( $17 \%$ increase). The other groups did not display any of the described effects or any other corridor interaction effects.

Interactions between matrix quality, environmental stress, and disturbance

Oribatid morphospecies richness decreased in landscapes with gravel matrix, but here the response to

TABLE 2. Summary table of treatment effects on abundance and species richness in the fragmented micro-ecosystem landscapes.

\begin{tabular}{|c|c|c|c|c|c|}
\hline Taxonomic group & Disturbance & Corridors & $\begin{array}{l}\text { Low matrix } \\
\text { quality }\end{array}$ & $\begin{array}{c}\text { High environmental } \\
\text { stress }\end{array}$ & Interactions \\
\hline Adult oribatid mites & negative & negative & negative & negative & disturbance $\times$ corridors \\
\hline Juvenile oribatid mites & negative & & & negative & $\begin{array}{l}\text { matrix } \times \text { environment } \\
\text { disturbance } \times \text { environment }\end{array}$ \\
\hline Oribatid species richness & negative & & & & matrix $\times$ environment \\
\hline Collembola & negative & & negative & & matrix $\times$ corridors \\
\hline Predators & negative & & negative & negative & $\begin{array}{l}\text { disturbance } \times \text { environment } \\
\text { disturbance } \times \text { corridors } \\
\text { disturbance } \times \text { matrix } \\
\text { matrix } \times \text { corridors }\end{array}$ \\
\hline
\end{tabular}

Notes: Effects with weak evidence and inconsistent main effects are excluded. See the Appendix for model-averaged parameter estimates. 

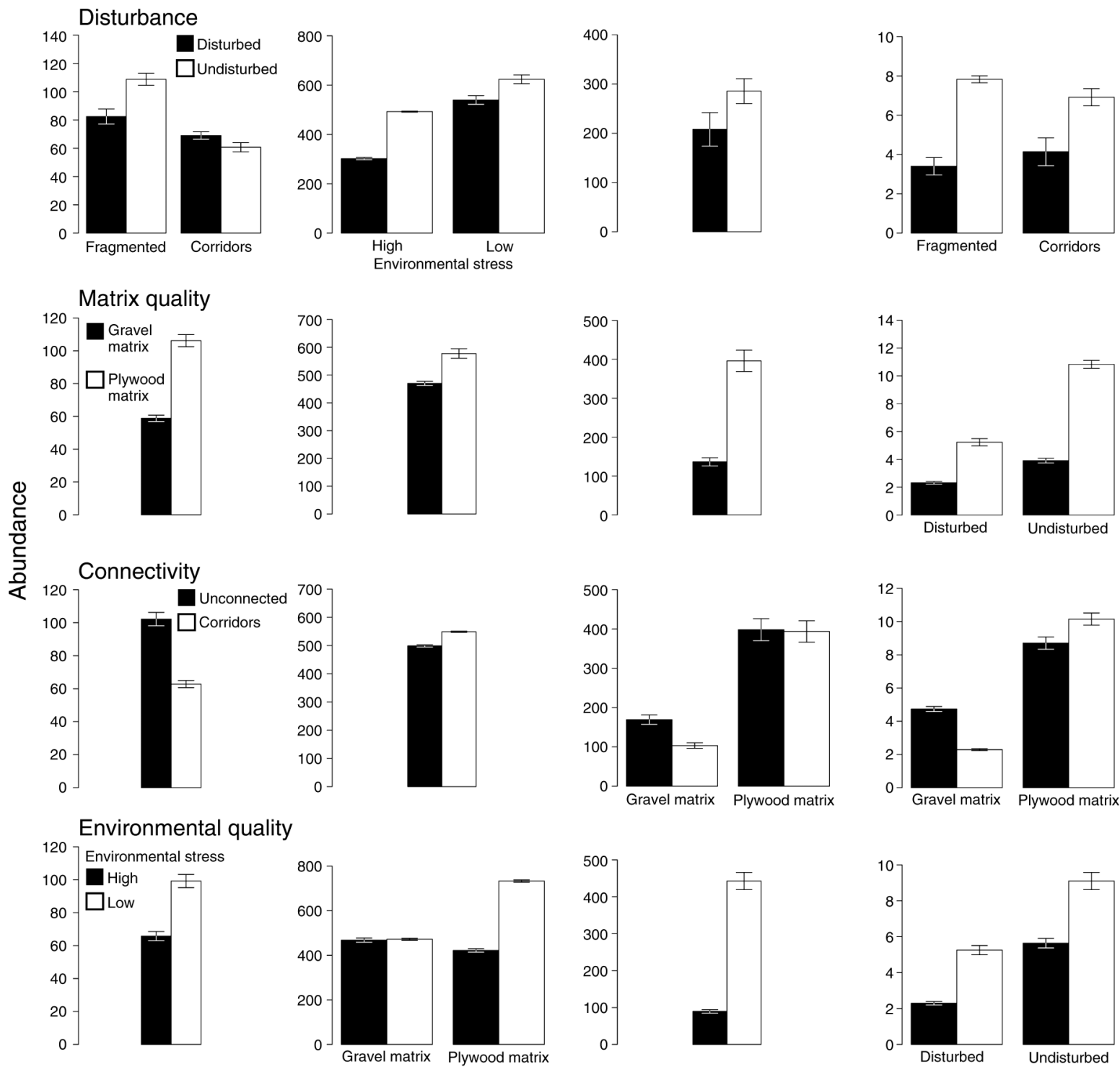

Adult oribatid mites

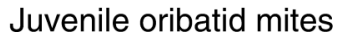

\section{Collembola}

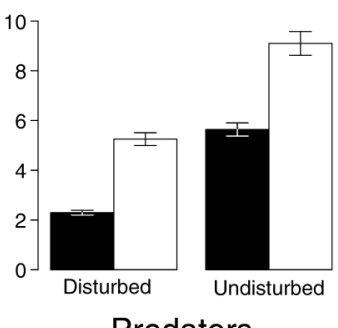

FIG. 2. Summary of treatment effects on abundance, based on unconditional predicted means of a balanced data set. Bars show abundances per local patch (mean \pm 2 SD) at the end of the experiment. Experimental landscapes consisted of four $100-\mathrm{cm}^{2}$ patches of moss, containing a rich ecosystem of microarthropods. Habitat patches were either disturbed initially by removal of individuals or undisturbed, were either connected through dispersal corridors or unconnected, were enclosed by a landscape matrix of either plywood or gravel, and were subjected to either natural desiccation events or regular watering.

matrix quality depended on environmental stress. In landscapes with high environmental stress (untreated) and gravel matrix, oribatid morphospecies richness decreased to $\sim 82 \%$ of that in landscapes with plywood matrix. In landscapes with low environmental stress, however, there was practically no difference in oribatid morphospecies richness due to matrix quality (Fig. 3). Thus, it seemed that both a gravel matrix and stressful environmental conditions were needed to reduce oribatid morphospecies richness. The influence of matrix quality on juvenile oribatid abundance also depended on environmental stress. Juvenile oribatid abundances were roughly equal, irrespective of matrix quality, except in landscapes with both plywood matrix and low environmental stress, where the numbers increased by roughly $55 \%$ (Fig. 2). Therefore, both plywood matrix and decreased environmental stress were necessary to increase juvenile oribatid abundance. Juvenile oribatids also showed larger declines due to disturbance in landscapes with high environmental stress than in landscapes with more benevolent conditions $(39 \%$ and $13 \%$ reduction in disturbed patches, respectively).

Matrix quality strongly influenced predator abundance, and in addition to the interaction effect with 

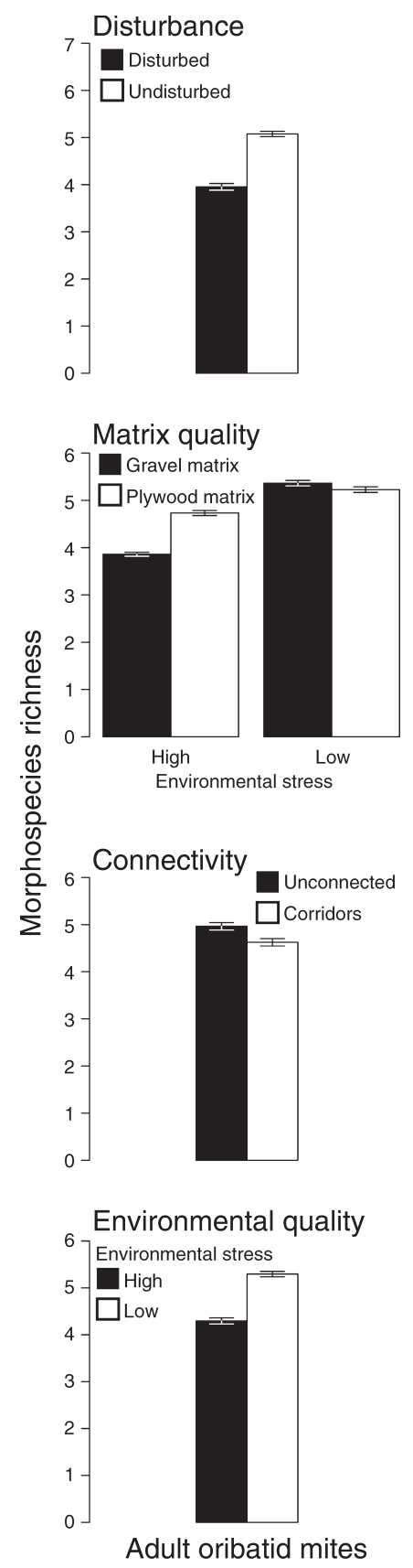

FIG. 3. Summary of treatment effects on oribatid morphospecies richness, based on unconditional predicted means of a balanced data set. Bars show morphospecies richness per local patch (mean $\pm 2 \mathrm{SD}$ ) at the end of the experiment. Experimental landscapes consisted of four $100-\mathrm{cm}^{2}$ patches of moss, containing a rich ecosystem of microarthropods. Habitat patches were either disturbed initially by removal of individuals or undisturbed, were either connected through dispersal corridors or unconnected, were enclosed by a landscape matrix of either plywood or gravel, and were subjected to either natural desiccation events or regular watering. connectivity, it also interacted with disturbance. The decrease in predator abundance due to matrix quality was more pronounced in undisturbed patches than in disturbed patches (64\% and 56\%, respectively). Lastly, there was also evidence of an interaction effect between disturbance and environmental quality on predator abundance. Consistent with the higher abundances of predators in the undisturbed patches, the negative effect of the environmental stress was also more pronounced there $56 \%$ reduction and $38 \%$ reduction in disturbed patches with high and low environmental stress, respectively).

\section{DisCUSSION}

The results supported some of our predictions and contradicted others. In line with the predictions, we found that local disturbance, gravel matrix, and increased environmental stress caused declines in the abundance of all examined species groups, as well as oribatid morphospecies richness (except for environmental stress). However, contrary to predictions, the effects of fragmentation were small and had a tendency for positive responses. In addition, corridors had negative effects on the abundance of oribatids, as well as collembolans and predators when the landscapes contained gravel matrix.

The only taxa that showed positive response to corridors were predators, conforming to the predicted sensitivity to fragmentation of organism groups with low abundance and high trophic rank (Gilbert et al. 1998, Davies et al. 2000, Östman et al. 2007). Although we found evidence that spatial structure mattered, and that the effects of spatial structure indeed interacted with the other treatments, several of these interactions were unexpected and may therefore increase the difficulty in predicting responses to landscape change. There was evidence that the effect of spatial structure is dependent on disturbance, identity of organism group, matrix quality, and environmental stress. Therefore, a main result of this study is that the response to spatial structure is context dependent, and that the end result of interlinking habitat fragments by dispersal corridors can be counterintuitive.

\section{Spatial structure and disturbance}

Potential interactions between fragmentation and disturbance are important because fragmentation and disturbance often co-occur (Lindenmayer and Fischer 2006). In addition, interactions between fragmentation and disturbance are expected because disturbed systems often require recolonization from surrounding undisturbed sources, and fragmentation naturally limits such recolonization (Bengtsson et al. 2003, Starzomski and Srivastava 2007). In our study, increasing the connectivity with habitat corridors decreased the difference in abundance between disturbed and undisturbed patches for oribatids and predators. However, corridors did not universally lead to increased abundances in the dis- 
turbed patches. Oribatid abundances became more similar but decreased overall in connected landscapes, while predator abundances became more similar and increased in the disturbed patches (Fig. 2). This is one of several examples in the present study of taxon-specific responses to dispersal corridors, emphasizing the potential for landscape structure to affect community composition.

The groups that did not display an interaction between corridors and disturbance (juvenile oribatids and collembolans) were less affected by corridors altogether (except for a corridor $\times$ matrix interaction). It is not surprising that the highly dispersive collembolans did not require corridors to cross these distances and therefore did not display a disturbance $\times$ corridor interaction. Less obvious is the relatively small influence of corridors on juvenile oribatids. Altogether, it seems that the total number of juvenile oribatids was not limited by dispersal in this study. This conforms to earlier studies (Åström and Bengtsson 2011) that suggested an increased reproductive rate in defaunated patches. Juveniles counts are influenced by both active dispersal and local egg hatchings, and the two mechanisms are not separately identifiable in this study. It is plausible, however, that substantial proportions of the juveniles in the disturbed patches were transported there as eggs, and that local reproduction is mainly limited by local habitat resources. Many of the oribatid species are parthenogenetic (Walter and Proctor 1999) and perhaps full recolonization of adults is not needed to saturate a disturbed local patch with juveniles.

\section{Negative effects of corridors}

The potential beneficial outcome of habitat corridors has long been a contested issue (Simberloff and Cox 1987, Noss and Beier 2000, Haddad and Tewksbury 2006). Recent examples of positive effects (Gilbert et al. 1998, Damschen et al. 2006), no effects (Hoyle and Gilbert 2004, Rantalainen et al. 2004), and negative effects (Matthiessen et al. 2010) of increased connectivity and dispersal highlight the complexity of the issue. One of the most interesting results of our experiment was the strong negative response of oribatid abundance to corridors, contrary to our expectations. In addition, corridors also had negative effects on collembolans and predators when the matrix was made of gravel. This suggests that corridors increase mortality or in other ways drain habitat patches of individuals, at least when the matrix has a more complex structure (gravel, in this case). We can identify four nonexclusive, theoretically possible explanations for such drainage of individuals in the presence of corridors, of which the first two are more likely than the others: increased dispersal into the matrix, increased mortality due to desiccation, increased competition, and increased predation.

Firstly, adding dispersal corridors by necessity increases the contact area to the surrounding matrix, and adding departure routes into the matrix might decrease abundances in the habitat patches. Especially if there generally is little need of increased inter-patch dispersal, as the lack of fragmentation effects in this study implies, adding corridors might have overall detrimental effects. However, this mechanism does not explain why collembolans and predators responded differently to corridors in landscapes with plywood matrix compared to landscapes with gravel matrix.

Secondly, corridors could have affected the microclimate in the patches. For example, Chisholm et al. (2011) showed significant "wicking effects" from corridors in similar settings, leading to desiccated patches. The generally strong effects of environmental stress suggest that desiccation indeed was a major mortality factor in our experiment. This mechanism implies interactions between corridors and environmental stress, which was observed for predators, and interactions between corridors and gravel matrix because of the heat from the sun-warmed gravel, which was observed for collembolans and predators.

Thirdly, connecting habitat patches, thereby increasing inter-patch dispersal, could under some conditions increase the chances of competitive exclusion (Loreau and Mouquet 1999). Because there were limited chances of reproduction in the present study due to the short time frame, competitive exclusion of species could potentially also lead to decreased abundances. However, $\beta$-diversity did not decrease in connected landscapes, nor did we observe any patterns of competitive exclusion among oribatid mites when comparing their responses species by species (not shown, see Methods), so this explanation appears unlikely.

Lastly, increased predation by the arthropod predators is also unlikely for several reasons. First, we found no general relationship between predator abundance and prey species abundance, with the possible exception of predators in landscapes with plywood matrix (cf. adult oribatids and predators; Fig. 2). Furthermore, at least many of the adult oribatids are heavily armored and thus the predation rate on adults is usually low (Peschel et al. 2006, Schneider and Maraun 2009). To sum up, our data suggest that corridors may have negative effects on abundance in different species groups, at least partially because of an increased mortality due to detrimental edge effects or by increased movements into a harsh matrix.

\section{Spatial structure, matrix quality, disturbance, and environmental stress}

Clearly, matrix quality may have profound effects on fragmented populations and communities (Figs. 2 and 3). Although matrix quality historically has been neglected in discussions of fragmentation, it is being increasingly discussed (see e.g., Vandermeer and Carvajal 2001, Jules and Shahani 2003). Attention has been given to whether the matrix offers resources and how matrix quality affects movement behavior across habitat 
edges (Haynes and Cronin 2006, Kupfer et al. 2006, Schmidt et al. 2008, Perfecto and Vandermeer 2010).

In line with our predictions, abundances of most species groups were higher in the landscapes with plywood matrix compared to the gravel matrix, suggesting that gravel matrix increased the drainage of individuals in the landscapes, possibly due to increased mortality and reduced permeability. Gravel matrix also interacted negatively with dispersal corridors for collembolans and predators, further exacerbating their decreased abundances in gravel matrix landscapes. Although unexpected (e.g., Öckinger and Smith [2008] found that corridors were more effective with less permeable matrix), this conforms with Baum et al. (2004), who found increased effectiveness of corridors with increased matrix permeability, although the mechanisms are probably not the same. Clearly, the interactions between matrix structure and quality and corridors may be complex, and we need more studies investigating the effects of corridors on movements both within and outside corridors.

Increasing connectivity could both prevent local extinctions and help to recolonize patches that have suffered local extinctions (Brown and Kodric-Brown 1977). In line with this reasoning, we expected landscapes with harsher environmental conditions to suffer more local extinctions and thus benefit more from corridors, which would manifest as an interaction between corridors and environmental stress. But as previously noted, we found no clear positive effects of corridors and thus the prerequisite for the expectation disappeared. Consequently, we did not find any interactions between environmental stress and corridors despite the generally strong negative effects of environmental stress (Figs. 2 and 3). Thus, this experiment demonstrates that microarthropod communities in moss patches have a general sensibility to drought, and, in particular, that environmental conditions can be influential also at relatively short time scales.

\section{Conclusions}

This study provides experimental evidence that dispersal corridors can negatively affect populations in fragmented metacommunities. This, and the lack of negative general effects of fragmentation, were contrary to our hypotheses and expectations, based on earlier reports from similar model systems (Gonzalez et al. 1998, Gonzalez and Chaneton 2002). For some species groups, the negative effect of dispersal corridors was contingent on matrix quality. This suggests that there might be a cutoff in matrix quality where the creation of corridors no longer increases the effective dispersal between patches. The outcome of connecting fragmented habitat patches by corridors will then be dependent on the interactions between the respective dispersal abilities in matrix and corridors, and the severity of the drainage effects of corridors in harsh environments.
Matrix quality therefore appears as a key factor to consider when planning dispersal corridors.

More expected, this study also shows that the consequences of disturbance may be dependent on landscape connectivity. Dispersal corridors were a positive factor for predator recovery after disturbance (cf. Starzomski and Srivastava 2007). This highlights the sensitivity of fragmented landscapes to disturbance and calls for the consideration of disturbance regimes in the management of spatially structured landscapes (Bengtsson et al. 2003).

Lastly, the influence of spatial structure appears to be dependent on specific attributes of the organism groups, such as dispersal ability and trophic level (see also Davies et al. 2000). This highlights that spatial structure may have varying influence on different parts of the community, and therefore may change community composition and species dominance (Crist et al. 2005, Cadotte 2006).

The results of this study warn against treating habitat corridors as a panacea to current fragmentation and encroachment problems. Instead, future theoretical and empirical studies of metacommunity dynamics need to consider interactions between landscape elements as well as environmental conditions and disturbance regimes in order to predict community changes in dynamic, spatially structured landscapes.

\section{ACKNOWLEDGMENTS}

We are grateful for the valuable input provided by Jan Bengtsson while we were designing the experiment and writing this paper. We thank Ola Bengtsson, Marie Melander, and Kristyna Müller for help with sample processing. The study was funded by the Swedish Research Council (grants to J. Bengtsson and T. Part).

\section{Literature Cited}

Anderson, M. J. 2001. Permutation tests for univariate or multivariate analysis of variance and regression. Canadian Journal of Fisheries and Aquatic Sciences 58:626-639.

Åström, J., and J. Bengtsson. 2011. Patch size matters more than dispersal distance in a mainland-island metacommunity. Oecologia 167:747-757.

Auclerc, A., J. Ponge, S. Barot, and F. Dubs. 2009. Experimental assessment of habitat preference and dispersal ability of soil springtails. Soil Biology and Biochemistry 41:1596-1604.

Bates, D., and M. Maechler. 2010. lme4: Linear mixed-effects models using S4 classes. The $\mathrm{R}$ Project for Statistical Computing. http://cran.r-project.org/package $=1 m e 4$

Baum, K., K. Haynes, F. Dillemuth, and J. Cronin. 2004. The matrix enhances the effectiveness of corridors and stepping stones. Ecology 85:2671-2676.

Bengtsson, J., P. Angelstam, T. Elmqvist, U. Emanuelsson, C. Folke, M. Ihse, F. Moberg, and M. Nyström. 2003. Reserves, resilience and dynamic landscapes. AMBIO 32:389-396.

Bolker, B. M., M. E. Brooks, C. J. Clark, S. W. Geange, J. R. Poulsen, M. H. H. Stevens, and J. S. White. 2009. Generalized linear mixed models: a practical guide for ecology and evolution. Trends in Ecology and Evolution 24:127-135.

Brown, J., and A. Kodric-Brown. 1977. Turnover rates in insular biogeography: effect of immigration on extinction. Ecology 58:445-449. 
Cadotte, M. 2006. Dispersal and species diversity: a metaanalysis. American Naturalist 167:913-924.

Chisholm, C., Z. Lindo, and A. Gonzalez. 2011. Metacommunity diversity depends on connectivity and patch arrangement in heterogeneous habitat networks. Ecography 34:415424.

Cottenie, K. 2005. Integrating environmental and spatial processes in ecological community dynamics. Ecology Letters 8:1175-1182.

Crist, T. O., S. V. Pradhan-Devare, and K. S. Summerville. 2005. Spatial variation in insect community and species responses to habitat loss and plant community composition. Oecologia 147:510-521.

Damschen, E. I., N. M. Haddad, J. L. Orrock, J. J. Tewksbury, and D. J. Levey. 2006. Corridors increase plant species richness at large scales. Science 313:1284.

Davies, K. F., C. R. Margules, and J. F. Lawrence. 2000. Which traits of species predict population declines in experimental forest fragments? Ecology 81:1450-1461.

Elston, D., R. Moss, T. Boulinier, C. Arrowsmith, and X. Lambin. 2001. Analysis of aggregation, a worked example: numbers of ticks on red grouse chicks. Parasitology 122:563569.

Gilbert, F., A. Gonzalez, and I. Evans-Freke. 1998. Corridors maintain species richness in the fragmented landscapes of a microecosystem. Proceedings of the Royal Society B 265:577.

Gonzalez, A., and E. J. Chaneton. 2002. Heterotroph species extinction, abundance and biomass dynamics in an experimentally fragmented microecosystem. Journal of Animal Ecology 71:594-602.

Gonzalez, A., J. H. Lawton, F. S. Gilbert, T. M. Blackburn, and I. Evans-Freke. 1998. Metapopulation dynamics, abundance, and distribution in a microecosystem. Science 281:2045.

Gravel, D., N. Mouquet, M. Loreau, and F. Guichard. 2010. Patch dynamics, persistence, and species coexistence in metaecosystems. American Naturalist 176:289-302.

Haddad, N., and J. Tewksbury. 2006. Impacts of corridors on populations and communities. Pages $390-415$ in K. Crooks and M. Sanjayan, editors. Connectivity conservation. Cambridge University Press, Cambridge, UK.

Hanski, I. 1999. Metapopulation ecology. Oxford University Press, Oxford, UK.

Haynes, K., and J. Cronin. 2006. Interpatch movement and edge effects: the role of behavioral responses to the landscape matrix. Oikos 113:43-54.

Hinde, J. 1982. Compound Poisson regression models. Pages 109-121 in R. Gilchrist, editor. GLIM 82, Proceedings of the International Conference on Generalised Linear Models. Springer-Verlag, Berlin, Germany.

Holt, R. D., J. H. Lawton, G. A. Polis, and N. D. Martinez. 1999. Trophic rank and the species-area relationship. Ecology 80:1495-1504.

Holyoak, M., M. A. Leibold, and R. D. Holt. 2005. Metacommunities: spatial dynamics and ecological communities. University of Chicago Press, Chicago, Illinois, USA.

Howeth, J. G., and M. A. Leibold. 2010. Prey dispersal rate affects prey species composition and trait diversity in response to multiple predators in metacommunities. Journal of Animal Ecology 79:1000-1011.

Hoyle, M., and F. Gilbert. 2004. Species richness of moss landscapes unaffected by short-term fragmentation. Oikos 105:359-367.

Hoyle, M., and A. R. Harborne. 2005. Mixed effects of habitat fragmentation on species richness and community structure in a microarthropod microecosystem. Ecological Entomology 30:684-691.

Hutson, B. R. 1980. Colonization of industrial reclamation sites by acari, collembola and other invertebrates. Journal of Applied Ecology 17:255-275.
Jules, E., and P. Shahani. 2003. A broader ecological context to habitat fragmentation: Why matrix habitat is more important than we thought. Journal of Vegetation Science 14:459464.

Kupfer, J. A., G. P. Malanson, and S. B. Franklin. 2006. Not seeing the ocean for the islands: the mediating influence of matrix-based processes on forest fragmentation effects. Global Ecology and Biogeography 15:8-20.

Leibold, M. A., M. Holyoak, N. Mouquet, P. Amarasekare, J. M. Chase, M. F. Hoopes, R. D. Holt, J. B. Shurin, R. Law, D. Tilman, M. Loreau, and A. Gonzalez. 2004. The metacommunity concept: a framework for multi-scale community ecology. Ecology Letters 7:601-613.

Lindberg, N., and J. Bengtsson. 2006. Recovery of forest soil fauna diversity and composition after repeated summer droughts. Oikos 144:494-506.

Lindberg, N., J. Engtsson, and T. Persson. 2002. Effects of experimental irrigation and drought on the composition and diversity of soil fauna in a coniferous stand. Journal of Applied Ecology 39:924-936.

Lindenmayer, D., and J. Fischer. 2006. Habitat fragmentation and landscape change: an ecological and conservation synthesis. Island Press, Washington, D.C., USA.

Loreau, M., and N. Mouquet. 1999. Immigration and the maintenance of local species diversity. American Naturalist 154:427-440.

Luxton, M. 1981. Studies on the oribatid mites of a Danish beech wood soil. Pedobiologia 21:312-340.

Matthiessen, B., E. Mielke, and U. Sommer. 2010. Dispersal decreases diversity in heterogeneous metacommunities by enhancing regional competition. Ecology 91:2022-2033.

Noss, R., and P. Beier. 2000. Arguing over little things: response to Haddad et al. Conservation Biology 14:15461548 .

Öckinger, E., R. Lindborg, E. Sjödin, and R. Bommarco. 2011. Landscape matrix modifies richness of plants and insects in grassland fragments. Ecography 35:259-267.

Öckinger, E., and H. G. Smith. 2008. Do corridors promote dispersal in grassland butterflies and other insects? Landscape Ecology 23:27-40.

Östman, Ö., N. W. Griffin, J. L. Strasburg, J. A. Brisson, A. R. Templeton, T. M. Knight, and J. M. Chase. 2007. Habitat area affects arthropod communities directly and indirectly through top predators. Ecography 30:359-366.

Perfecto, I., and J. Vandermeer. 2010. The agroecological matrix as alternative to the land-sparing/agriculture intensification model. Proceedings of the National Academy of Sciences USA 107:5786.

Peschel, K., R. A. Norton, S. Scheu, and M. Maraun. 2006. Do oribatid mites live in enemy-free space? Evidence from feeding experiments with the predatory mite Pergamasus septentrionalis. Soil Biology and Biochemistry 38:2985-2989.

R Development Core Team. 2010. R: A Language and Environment for Statistical Computing. R Foundation for Statistical Computing, Vienna, Austria. http://www. r-project.org

Rantalainen, M., J. Haimi, H. Fritze, and H. Setala. 2006. Effects of small-scale habitat fragmentation, habitat corridors and mainland dispersal on soil decomposer organisms. Applied Soil Ecology 34:152-159.

Rantalainen, M., J. Haimi, and H. Setala. 2004. Testing the usefulness of habitat corridors in mitigating the negative effects of fragmentation: the soil faunal community as a model system. Applied Soil Ecology 25:267-274.

Remén, C., M. Krüger, and A. Cassel-Lundhagen. 2010. Successful analysis of gut contents in fungal-feeding oribatid mites by combining body-surface washing and PCR. Soil Biology and Biochemistry 42:1952-1957.

Schmidt, M., C. Thies, W. Nentwig, and T. Tscharntke. 2008. Contrasting responses of arable spiders to the landscape 
matrix at different spatial scales. Journal of Biogeography 35:157-166.

Schneider, K., and M. Maraun. 2009. Top-down control of soil microarthropods - evidence from a laboratory experiment. Soil Biology and Biochemistry 41:170-175.

Siepel, H. 1994. Life-history tactics of soil microarthropods. Biology and Fertility of Soils 18:263-278.

Simberloff, D., and J. Cox. 1987. Consequences and costs of conservation corridors. Conservation Biology 1:63-71.

Srivastava, D. 2004. Are natural microcosms useful model systems for ecology? Trends in Ecology and Evolution 19:379-384.

Starzomski, B. M., and D. S. Srivastava. 2007. Landscape geometry determines community response to disturbance. Oikos 116:690-699.
Vandermeer, J., and R. Carvajal. 2001. Metapopulation dynamics and the quality of the matrix. American Naturalist 158:211-220.

Vanschoenwinkel, B., A. Waterkeyn, M. Jocqué, L. Boven, M. Seaman, and L. Brendonck. 2010. Species sorting in space and time - the impact of disturbance regime on community assembly in a temporary pool metacommunity. Journal of the North American Benthological Society 29:1267-1278.

Venables, W. N., B. D. Ripley, and W. N. Venables. 1998. Modern applied statistics with S-Plus. Second edition. Springer-Verlag, Berlin, Germany.

Walter, D., and H. Proctor. 1999. Mites: ecology, evolution and behaviour. University of New South Wales Press, Sydney, Australia.

\section{Supplemental Material}

\section{Appendix}

Summary of experimental units, summary of identified taxonomic units and number of individuals, and parameter estimates tables (Ecological Archives E094-008-A1). 